\title{
Kalman filter estimation of RLC parameters for UMP transmission line
}

\author{
Siti Nur Aishah Mohd Amin, Hamzah Ahmad and Mohd Rusllim Mohamed \\ Faculty of Electrical \& Electronics, Universiti Malaysia Pahang, 26600 Pekan, Pahang
}

\begin{abstract}
This paper present the development of Kalman filter that allows evaluation in the estimation of resistance (R), inductance (L), and capacitance (C) values for Universiti Malaysia Pahang (UMP) short transmission line. To overcome the weaknesses of existing system such as power losses in the transmission line, Kalman Filter can be a better solution to estimate the parameters. The aim of this paper is to estimate RLC values by using Kalman filter that in the end can increase the system efficiency in UMP. In this research, matlab simulink model is developed to analyse the UMP short transmission line by considering different noise conditions to reprint certain unknown parameters which are difficult to predict. The data is then used for comparison purposes between calculated and estimated values. The results have illustrated that the Kalman Filter estimate accurately the RLC parameters with less error. The comparison of accuracy between Kalman Filter and Least Square method is also presented to evaluate their performances.
\end{abstract}

\section{Introduction}

Electrical energy is generated at power stations which are typically located far away from the load centres [1]. Thus, a network of conductors between the power stations and the consumers is required in order to utilize the power generated. This network of conductors is divided into two main components, which are the transmission system and the distribution system. At a generating plant, electric power is "stepped up" to several thousand volts by a transformer and delivered to the transmission line. At numerous substations on the transmission system, transformers step down the power to a lower voltage and deliver it to the different load centres in the distribution lines [2]. This is because high voltage improves efficiency by reducing heat loss in the power line. But high voltages are too dangerous, so step down transformers are used to reduce the voltage to a required and safe levels.

The power transmission line is one of the major components in electrical power system. All transmission lines in a power system posses the electrical properties of resistance, inductance and capacitance [3]. These passive elements are usually referred to investigate and assess the transmission line characteristics and efficiency which makes them irresistible to be analysed as part of system performance. The series resistance relies basically on the physically composition of the conductor at a given temperature. While the series inductance and capacitance is produced by the presence of magnetic field and electric field around the conductors. Besides, the series inductance and capacitance depends on the geometrical arrangement in a transmission line [4]. The important consideration in the design and operation of transmission line are the determination of voltage drop, line losses and efficiency of transmission line. These values are greatly influenced by the constant resistance (R), inductance (L) and capacitance (C) in the transmission line [5]. According to the previous paper, resistance $(\mathrm{R})$, reactance $(\mathrm{X})$ and susceptance $(\mathrm{B})$ also affected the transmission line performance in a system [3]. The frequency involve in this three constant parameter indicates the performance of a system. When considering high frequency, the magnetic field generated drive the currents to the outer edge of the conductor that carries them. As a result, the higher the frequency, the thinner the layer of metal available to carry the current and the higher the effective resistance of the cable [6]. In fact, the voltage drop in the line depends on the values of above three lines constant [7]. In addition, the resistance of transmission line is the most important cause of power losses in the line and it determines the transmission line efficiency. Since there are many research has been done on $\mathrm{R}, \mathrm{X}$ and $\mathrm{B}$ values in medium transmission line, so this paper will focuses on $\mathrm{R}, \mathrm{L}$ and $\mathrm{C}$ values in UMP short transmission line. This is because of calculation of power factor relies on these parameters.

Power system state estimation is a main tool of energy management system. State estimation was first applied to power systems by Schweppe and Wildes in the late 1960s [8][9]. Since then, several applications and techniques of Kalman filter have been one of the famous methods in power system estimation. It has been used for damping and frequency [7], modal changes [10], state estimation [11], power factor detection and etc. However all of these researches did not highlighted important indicators that give great effects in the system which are RLC parameters. Most of these papers are focused solely

\footnotetext{
* Corresponding author: aishahamin 92@yahoo.com
} 
on IEEE system and are not tested on a real system. One of the systems that have been tested is long transmission line [12]. Theoretically, values of $\mathrm{R}, \mathrm{L}$ and $\mathrm{C}$ in long transmission line are supposedly higher than short transmission line. But due to some condition, the values of $\mathrm{R}, \mathrm{L}$ and $\mathrm{C}$ can be opposed the actual properties such as type of cable, size of cable, length of cable, number of winding and etc. All of these properties will indicate the values of $R, L$ and $C$ in the transmission line.

Since the real situation is different from the theoretical analysis due to unexpected condition such as the presence of noise and system dynamics, practical analysis in a real system also one of the essential aspects to be investigated to understand how the system behaves. In fact, by using a real system for analysis purposes, certain parameters can be determine such as the power losses, fault, and voltage drop. Therefore, this will create an opportunity for researcher to provide a suitable corrective action according to the circumstances. As a result, the determination for the future condition in transmission line can be identified. If the theoretical analysis and the real system have produced similar results then the system physical properties such as type, size and length of cable can be determined more precisely by the load applied in the system.

An accurate understanding of transmission line parameters is necessary for accurate power flow modelling, fault location, transient stability and etc. Previously, calculations based in conductor dimensions, sag and line length were used to estimates these parameters [13]. However, due to the assumptions and approximation involved, the estimation was not reliable for real - time application [13]. Therefore, this situation has inspired this research to examine UMP transmission line as shown in Figure 1. It is also worth to mention that, currently UMP do not have any information about the parameters of the transmission line. This situation has produced difficulties to reduce the operating cost and maintenance operations. To overcome this issue, Kalman filter method is used for estimation of $\mathrm{R}, \mathrm{L}$ and $\mathrm{C}$ values for short transmission line in UMP. The analysis mainly investigates the $\mathrm{R}, \mathrm{L}$, and $\mathrm{C}$ parameters. Mathematical model for UMP short transmission line is designed by using standard parameters. Our analysis attempts to provide information for Department of Property Management and Development in UMP about the transmission line to understand the system performance as well as to execute a corrective action accordingly based on the given results. The Kalman filter model for estimation of UMP transmission line will be examined in the next section.

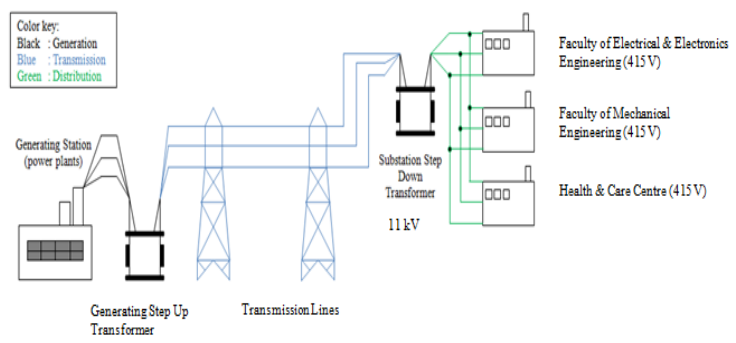

Fig. 1. Power distribution system in UMP

\section{Kalman filter method based on RLC estimation}

A transmission line can be defined as a short length line if its length is less than $50 \mathrm{~km}$ and the voltage is comparatively lower than $20 \mathrm{kV}$. In this case, the shunt capacitance (C) effect is negligible and only the resistance and inductive reactance are taken into account. Balanced conditions of the line can be represented by the equivalent circuit of a single phase with resistance (R), and inductance (L) in series (series impedance) [14], as shown in Figure 2.

If the transmission line has a length between $50 \mathrm{~km}$ and $150 \mathrm{~km}$ and the voltage is between $20 \mathrm{kV}-100 \mathrm{kV}$, the line is considered as a medium-length line and the equivalent circuit with resistance (R), inductance (L) and capacitance (C). Both short and medium length transmission lines use approximated lumped-parameter models [14] [15]. The lumped element model simplifies the description of the behaviour of spatially distributed physical systems into a topology consisting of discrete entities that approximate the behaviour of the distributed system under certain assumptions [5] [15].

However, if the transmission line is longer than 150 $\mathrm{km}$, and the voltage is higher than $100 \mathrm{kV}$, the model will be considered as a long-length line. The appropriate series impedance and capacitance are found by solving the corresponding differential equations, where voltages and currents are described as a function of distance and time [14]. As for our case, the short transmission line is considered for UMP.

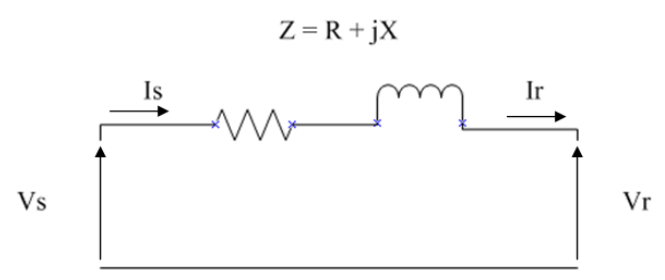

Fig. 2. Equivalent circuit for short transmission line

\section{A. Kalman Filter}

For a linear system, the process model of Kalman filter is described as

$$
\begin{gathered}
X_{k}=A X_{k-1}+B U_{k}+W_{k} \\
Z_{k}=H X_{k}+V_{k}
\end{gathered}
$$

where $X_{k}$ is the estimation value of $X, X_{k-1}$ is the estimate value on the previous state, $U_{k}$ is the control input, $W_{k}$ and $V_{k}$ is noise covariance matrix, $\mathrm{A}, \mathrm{B}$ and $\mathrm{C}$ will be numerical constant. and $\mathrm{C}$ as

The estimation value of $X_{k}$ is represented by $\mathrm{R}, \mathrm{L}$

$$
X_{k}=\left[\begin{array}{l}
R \\
L \\
C
\end{array}\right]
$$

where $R$ is resistance, $L$ is inductance and $C$ is capacitance. In this study, current, $A$ is used as a control input, $U_{k}$. 


$$
U_{k}=\left[\begin{array}{ccc}
A & 0 & 0 \\
0 & A & 0 \\
0 & 0 & A
\end{array}\right]
$$

The estimated value on the previous state, $X_{k-1}$ is represented by

$$
X_{k-1}=\left[\begin{array}{l}
R_{k-1} \\
L_{k-1} \\
C_{k-1}
\end{array}\right]
$$

\section{B. Prediction and update}

Kalman filter recursively computes estimation for a state $X_{k}$ which includes $\mathrm{R}, \mathrm{L}$ and $\mathrm{C}$ parameters according to the process and observation model [17]. The stages of Kalman filter algorithm are as follows:

- Prediction (time update) to estimate priori estimation of state and its error covariance matrix:

$$
\begin{aligned}
& X_{k}=A X_{k-1}+B U_{k} \\
& P_{k}=A P_{k-1} A^{T}+Q
\end{aligned}
$$

where $P_{k}$ is the prior error covariance. This prior value is used in the measurement update equations.

- Correction (measurement update) to provide correction based on the measurement $Z_{k}$ to yield the posteriori state estimate and its error covariance:

$$
\begin{gathered}
K_{k}=P_{k} H^{T}\left(H P_{k} H^{T}+R\right)^{-1} \\
X_{k}=X_{k}+K_{k}\left(Z_{k}-H X_{k}\right) \\
P_{k}=\left(1-K_{k} H\right) P_{k}
\end{gathered}
$$

where $K_{k}$ is the Kalman gain.

Figure 5 shows the the Kalman filter model for the UMP transmission line. The analysis mainly observes the RLC parameters based on different noise a condition which describes various unexpected issues during transmission in UMP. It is expected that from the proposed technique, UMP management is able to plan and execute suitable actions to gain the desired system performance.

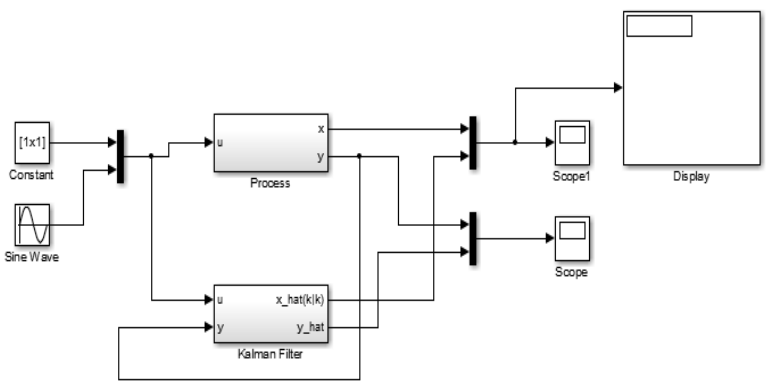

Fig. 5. Kalman filter model

\section{Theoretical analysis}

A $11 \mathrm{kV}, 5 \mathrm{~km}$ long, $60 \mathrm{~Hz}$ transmission line is considered in this research to model the UMP short transmission line. This section analyses the calculated $\mathrm{R}$, $\mathrm{L}$ and $\mathrm{C}$ parameters of the transmission line based on Figure 2 as this is the main reference for estimation purposes. Other quantities such as active power and impedance are calculated by using Ohms Law shown in below equations.

$$
\begin{gathered}
P=\sqrt{3} V I \cos \theta \\
\cos \theta=0.93 \\
V=I Z \\
Z=49.84 \Omega
\end{gathered}
$$

Figure 6 shows the power triangle characteristics which defined $\phi$ as the cosine of the angle between voltage and current phasor [18]. The angle of $\phi$ will be used to derive the value of $\mathrm{R}, \mathrm{L}$ and $\mathrm{C}$ of the short transmission line.

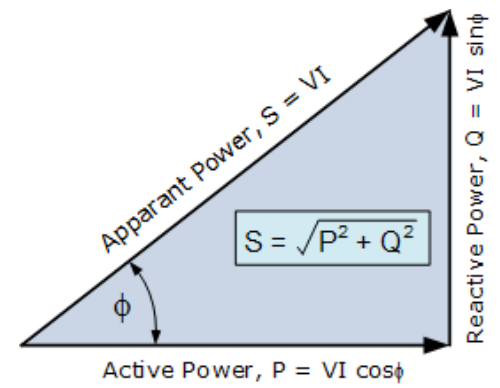

Fig. 6. Power triangle

$$
\begin{gathered}
p f=\cos \theta \\
p f=\frac{R}{Z}=\frac{P}{S} \\
p f=0.93 \\
R=46.35 \Omega
\end{gathered}
$$

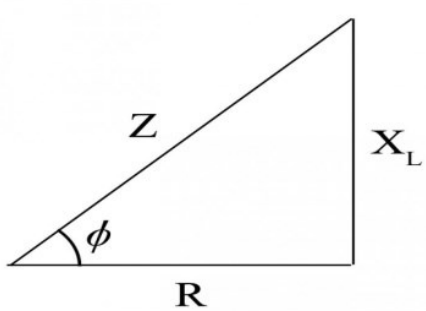

Fig. 7. Phasor diagram

From the phasor diagram above[18],

It is known that,

$$
\theta=\tan ^{-1}\left(\frac{\omega L}{R}\right)
$$

$$
\omega=2 \pi f
$$

Substituting all the related value into (17), the equation becomes,

$$
\omega=376.99
$$

By referring to equation (14), the value of $p f$ is substitute to get the value of $\theta$,

$$
\begin{aligned}
& \cos \theta=0.93 \\
& \theta=21.5652
\end{aligned}
$$

In order to get the value of $\mathrm{L}$, all the values are substitute into equation (16), and exhibit

$$
L=48.58 \mathrm{mH}
$$

As demonstrated above, the $\mathrm{R}, \mathrm{L}$ and $\mathrm{C}$ values have been calculated. The phase per metre line parameters $\mathrm{R}, \mathrm{L}, \mathrm{C}$ are $46.35 \Omega, 48.58 \mathrm{mH}$ and $0 \mathrm{~F}$ 
respectively. The line is operated in a power of $3911 \mathrm{~kW}$ at receiving end. These values will be assessed for estimation analysis covered in the next section.

\section{System model and analysis}

This subsection investigates the results of having different noise conditions to the estimation of UMP short transmission line. This paper takes into account system noises of 0.1 and 10 to simulate the unknown parameters which can effects the estimation results such as size, length and type of cables. Previous section has described how the value of $\mathrm{R}, \mathrm{L}$ and $\mathrm{C}$ are calculated. This section analyse how Kalman Filter works to estimate those value efficiently. The results obtained are described as follows.

Simulation was conducted for the equivalent circuit of short transmission line to simulate the UMP condition and tuning process also take place in order to achieve a good estimation results. Figure 8, Figure 9 and Figure 10 below shows the calculated and estimation values of $\mathrm{R}$, $\mathrm{L}$ and $\mathrm{C}$ over a period. The values of calculated $\mathrm{R}, \mathrm{L}$ and C simulated by using Kalman filter are $46.35 \Omega, 48.58$ $\mathrm{mH}$ and $0 \mathrm{~F}$ while the average values of estimated are $46.34 \Omega, 48.57 \mathrm{mH}$ and $0 \mathrm{~F}$ respectively with noise of 0.1 . There are small differences between the previous and new estimated values of $\mathrm{R}, \mathrm{L}$ and $\mathrm{C}$. Both of the calculated and estimated parameters are seen overlapping with each other due to the approximate parameter values. The result shows the higher consistency involved in $\mathrm{R}, \mathrm{L}$ and $\mathrm{C}$ value due to the approximate results between calculated and estimated values. According to Figure 10, the value of capacitance is $0 \mathrm{~F}$ as it is assumed negligible due to the properties of short transmission line. It must be noted that the initial values of estimation error covariance matrix affects the estimation of parameter values.

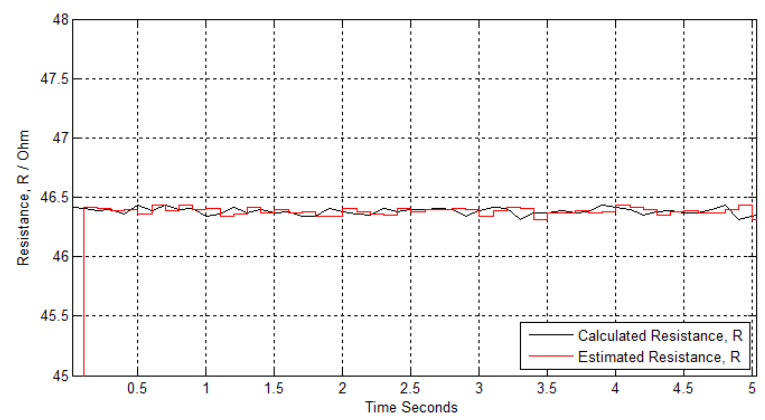

Fig. 8. Graph of calculated and estimated value of resistance, $R$

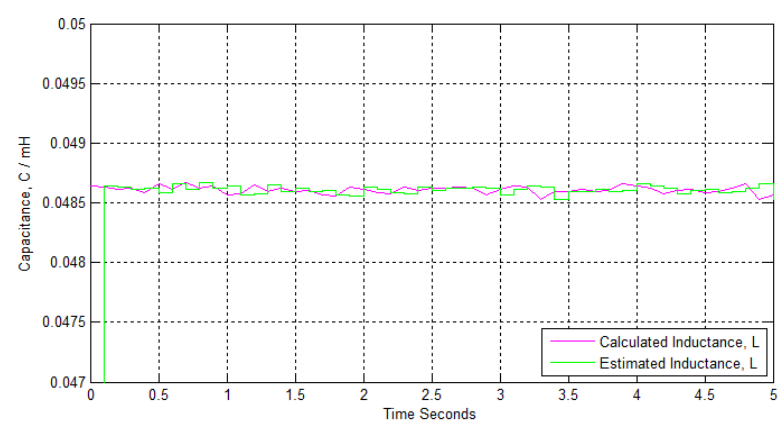

Fig. 9. Graph of calculated and estimated value of inductance, L

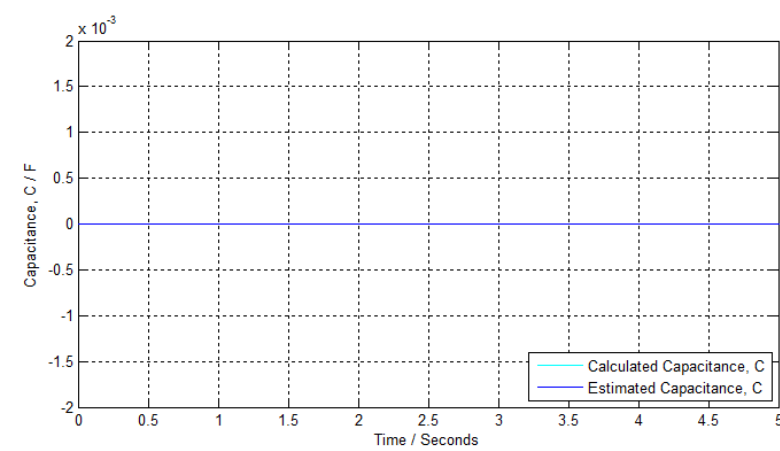

Fig. 10. Graph of calculated and estimated value of capacitance, $\mathrm{C}$

Figure 11, Figure 12 and Figure 13 below also shows the calculated and estimation values of $\mathrm{R}, \mathrm{L}$ and $\mathrm{C}$ in the system. The calculated values of $\mathrm{R}, \mathrm{L}$ and $\mathrm{C}$ are $46.35 \Omega, 48.58 \mathrm{mH}$ and $0 \mathrm{~F}$ while the estimated average values of $\mathrm{R}, \mathrm{L}$ and $\mathrm{C}$ are $46.22 \Omega, 48.43 \mathrm{mH}$ and $0 \mathrm{~F}$ respectively with higher system noise of 10 . Both of the calculated and estimated parameters are seen less consistency and the results are slightly different due to the greater noise in the system. Figure 13 shows the value of capacitance which is $0 \mathrm{~F}$.

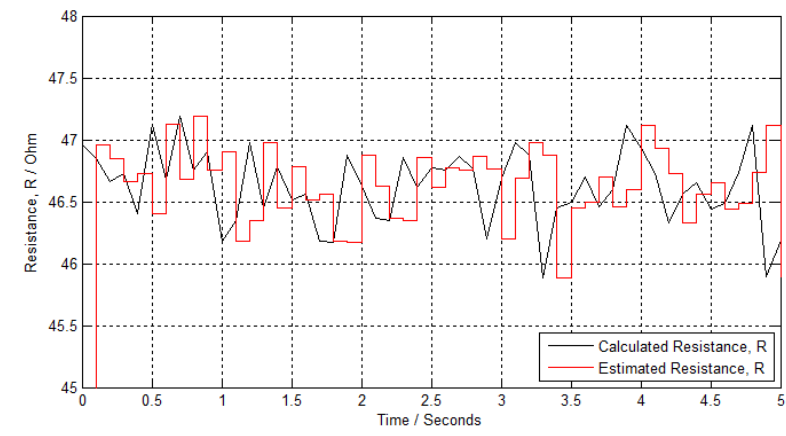

Fig. 11. Graph of calculated and estimated value of resistance, $\mathrm{R}$

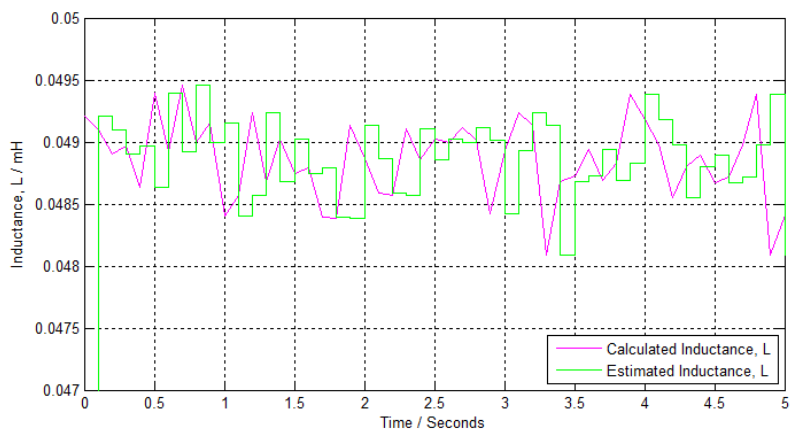

Fig. 12. Graph of calculated and estimated value of inductance, L 


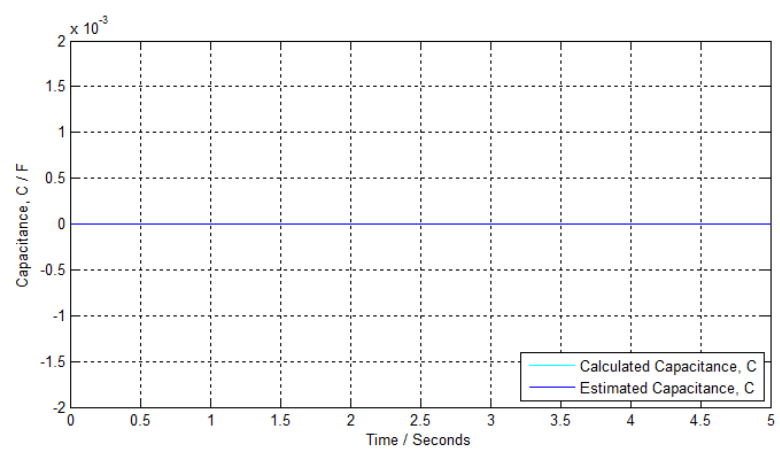

Fig. 13. Graph of calculated and estimated value of capacitance, $\mathrm{C}$

Table 1 defines the system parameters of the simulink model with the small value of noise which is 0.1 and 10. It shows the accuracy of resistance and inductance in the system more precise with the small value of noise which is $99.98 \%$ and $99.98 \%$ respectively. Thus, it is shown that higher estimation accuracy will be achieved with the decreasing values of the noise. This table also defines the system parameters of the simulink model with the greater value of noise which is 10 . It shows the accuracy of resistance and inductance in the system is inaccurate with the greater value of noise which is $99.72 \%$ and $99.69 \%$ respectively. Thus, it is proved that the accuracy in the system will decrease with the increasing noise.

Table 1. Parameters value with noise

\begin{tabular}{|c|c|c|c|}
\hline \multicolumn{5}{|c|}{ Noise $=0.1$} \\
\hline Parameter & $\begin{array}{c}\text { Calculated } \\
\text { value } \\
\text { (average) }\end{array}$ & $\begin{array}{c}\text { Estimation } \\
\text { value } \\
\text { (average) }\end{array}$ & Accuracy (\%) \\
\hline $\mathrm{R}$ & $46.35 \Omega$ & $46.34 \Omega$ & 99.98 \\
\hline $\mathrm{L}$ & $48.58 \mathrm{mH}$ & $48.57 \mathrm{mH}$ & 99.98 \\
\hline $\mathrm{C}$ & $0 \mathrm{~F}$ & $0 \mathrm{~F}$ & - \\
\hline $\mathrm{R}$ & $46.35 \Omega$ & $46.22 \Omega$ & 99.72 \\
\hline $\mathrm{L}$ & $48.58 \mathrm{mH}$ & $48.43 \mathrm{mH}$ & 99.69 \\
\hline $\mathrm{C}$ & $0 \mathrm{~F}$ & $0 \mathrm{~F}$ & - \\
\hline
\end{tabular}

According to this table and figures presented previously, the value of noise affected the accuracy of $\mathrm{R}$, $\mathrm{L}$ and $\mathrm{C}$ estimation in the system. The higher the value of noises or disturbance in the system, the performance degrades. These results also indicates the possibility to estimate the RLC parameters accurately even in different places especially when the same cable is being used. Higher voltage increased the noise problem in a system.
These situations occur due to the electron movement within the electrical circuits.

Comparison of accuracy between least square method and Kalman filter also has been done [3]. From Table 2 below, the results presented that Kalman filter give a higher accuracy compared to the Least Square method. Note that, Least Square method is used to estimate the values of $\mathrm{R}, \mathrm{X}$ and $\mathrm{B}$ in long transmission line located in East Midlands, England [3] while Kalman filter is used to indicates the values of $\mathrm{R}, \mathrm{L}$ and $\mathrm{C}$ in UMP. Eventhough the research is conducted in different places but the value of accuracy can still be taken into account. A better accuracy will come from short transmission line compared to long transmission line. This is due to the unknown noises which bigger system will have more unknown noises compared to the small system. From these two results of accuracy, it can be proven that Kalman filter is a better method that can be used for estimation.

Table 2. Parameters value

\begin{tabular}{|c|c|c|c|}
\hline \multicolumn{2}{|c|}{ Least Square method } & \multicolumn{2}{c|}{ Kalman filter } \\
\hline Parameter & Accuracy (\%) & Parameter & Accuracy (\%) \\
\hline R & 96.28 & R & 99.98 \\
\hline X & 99.97 & L & 99.98 \\
\hline B & 94.53 & C & - \\
\hline
\end{tabular}

Remark that, it is important to consider of $\mathrm{R}, \mathrm{L}$, and $\mathrm{C}$ and $\mathrm{R}, \mathrm{X}$ and $\mathrm{B}$ values as they affected the determination of power transfer, voltage drop and efficiency in the transmission line. The voltage drop in the line depends upon the values of above three lines constant. Similarly, the resistance of transmission line is the most important cause of power losses in the line and determine the transmission efficiency. Same as R, L and $\mathrm{C}$, values of $\mathrm{R}, \mathrm{X}$ and $\mathrm{B}$ also affected the determination of efficiency in a system.

\section{Conclusions}

This paper presented the analysis of $\mathrm{R}, \mathrm{L}$ and $\mathrm{C}$ estimation in a system by using Kalman filter. It was shown that although there is a little data from UMP short transmission line, the estimation of $\mathrm{R}, \mathrm{L}$ and $\mathrm{C}$ parameters is still possible. From this research, it has been revealed that noise in the system indicates the performance of UMP short transmission line and the parameter estimation can help the engineers in estimation for future growth even in a different area. In addition, with the different in accuracy according to the method used, this can be proven that Kalman filter is one of the optimal method that can be used for estimation for real - time line parameter estimator. 
Author would like to thank University Malaysia Pahang and Ministry of Higher Education for the supports in conducting the research under RDU160379 and RDU160145.

\section{References}

1. P. Leader and M. V Venkatasubramanian, "Coordination of Transmission Line Transfer Capabilities," 1996.

2. H. B. Harrison, "Transmission Line," no. May, pp. 111-113, 1982.

3. D. Ritzmann, P. S. Wright, W. Holderbaum, and B. Potter, "A Method for Accurate Transmission Line Impedance Parameter Estimation," IEEE Trans. Instrum. Meas., vol. 65, no. 10, pp. 2204-2213, 2016.

4. G. Chen and E. G. Friedman, "Effective Capacitance of RLC Loads for Estimating ShortCircuit Power," pp. 2065-2068, 2006.

5. S. Ma, Z. Bo, and A. Klimek, "The research on lumped parameter equivalent circuit of transmission line," pp. 2-6, 2009.

6. B. Curran, I. Ndip, and K. Lang, "A Comparison of Typical Surface Finishes on the High Frequency Performances of Transmission Lines in PCBs," pp. 1-3, 2017.

7. M. Larsson and C. Rehtanz, "Detection of Oscillations in Power Systems using Kalman Filtering Techniques,” pp. 183-188, 2013.

8. A. Saikia and R. K. Mehta, "Power system static state estimation using Kalman filter algorithm," 2016.
9. G. Gede, "Introduction to Kalman Filtering," Robotica, no. June, 2011.

10. R. A. Wiltshire, G. Ledwich, S. Member, and P. O. Shea, "A Kalman Filtering Approach to Rapidly Detecting Modal Changes in Power Systems," vol. 22, no. 4, pp. 1698-1706, 2007.

11. K. Vadirajacharya and B. A. T. U. Lonere, "Kalman filter analysis in dynamic state of power system," pp. 1170-1173, 2015.

12. E. C. M. Costa and S. Kurokawa, "Estimation of transmission line parameters using multiple methods," vol. 9, pp. 2617-2624, 2015.

13. C. Mishra and V. A. Centeno, "Kalman-Filter Based Recursive Regression for Three- Phase Line Parameter Estimation using Synchrophasor Measurements," 2015.

14. S. Gajare, A. K. Pradhan, and V. Terzija, "A Method for Accurate Parameter Estimation of Series Compensated Transmission Lines Using Synchronized Data," IEEE Trans. Power Syst., vol. 8950, no. c, pp. 1-1, 2017.

15. M. A. Shicong, G. U. O. Jianbo, S. U. N. Huadong, and Y. U. Yongjin, "The comparison and analysis of lumped parameter equivalent circuits of transmission line," 2011.

16. A. R. J. Araújo, R. C. Silva, and S. Kurokawa, "Comparing Lumped and Distributed Parameters Models in,” pp. 1-5, 2014.

17. G. Bishop and N. Carolina, "An Introduction to the Kalman Filter," 2001.

18. Ravish R. Singh, "Electrical Networks," vol. 352, no. August, pp. 35-42, 2003. 